\title{
Limitaciones del conectivismo en el Ecuador: necesidades urgentes para la calidad
}

\section{Limitations of connectivism in Ecuador: urgent needs for quality}

\author{
Christian Virgilio Guerrero Salazar ${ }^{1}$ \\ cvguerrero.est@uteg.edu.ec \\ https://orcid.org/0000-0003-3108-1846
}

Recibido: 28/9/2021, Aceptado: 28/12/2021

\begin{abstract}
RESUMEN
El presente estudio proyecta un análisis reflexivo de la situación actual de la educación en el Ecuador, y las limitaciones que ésta tiene para adaptarse a las nuevas realidades que exige la evolución constante de las tecnologías y el conectivismo, aceleradas en el último año debido a las necesidades surgidas por la pandemia del COVID 19 a nivel mundial. La propuesta de la investigación tiene un alcance descriptivo y es de tipo documental, ya que pretende analizar desde la perspectiva de varios autores, la problemática de la implementación de los modelos educativos basados en el conectivismo, en una realidad deficiente y poco preparada para los retos que impone un cambio paradigmático tan profundo. El estudio se enmarca en 3 aristas de análisis principales: la dificultad de acceso por razones económicas, el tradicionalismo de pensamiento colectivo y la falta de capacitación docente, tratando de establecer criterios que aporten a la comprensión crítica de estos procesos y su incidencia en la problemática investigada. Las conclusiones de la investigación, propenden a la búsqueda de soluciones que a corto o largo plazo permitan zanjar las brechas existentes para la implementación definitiva del conectivismo en el Ecuador, como opción imperante ante el desarrollo de la nueva sociedad del conocimiento y la búsqueda de la calidad educativa.
\end{abstract}

Palabras clave: conectivismo, modelos pedagógicos, pedagogía, calidad educativa

\section{ABSTRACT}

The present study projects a thoughtful analysis of the current situation of education in Ecuador, and the limitations that it has to adapt to the new realities required by the constant evolution of technologies and connectivism, accelerated in the past year due to the needs arising from the COVID 19 pandemic at the global level. The research proposal has a descriptive scope and is documentary, since it aims to analyze from the perspective of several authors, the problem of the implementation of educational models based on connectivism, in a deficient reality and unprepared for the challenges imposed

\footnotetext{
${ }^{1}$ Maestrando de la Universidad Tecnológica Empresarial de Guayaquil, UTEG
} 


\section{Revista Ciencia \& Tecnología $\begin{aligned} & \text { No. } 33,31 \text { de enero de } 2022 \\ & \text { ISSN impreso: } 1390 \text { - } 6321 \\ & \text { ISSN online: } 2661 \text { - } 6734\end{aligned}$}

by such a profound paradigm shift. The study is framed in 3 main areas of analysis: the difficulty of access for economic reasons, the traditionalism of collective thought and the lack of teacher training, trying to establish criteria that contribute to the critical understanding of these processes and their impact on the problem investigated. The conclusions of the research, tend to the search for solutions that in the short or long term allow to resolve the existing gaps for the definitive implementation of connectivism in Ecuador, as the prevailing option for the development of the new knowledge society and the search for educational quality.

Keywords: connectivism, pedagogical models, pedagogy, educational quality

\section{Introducción}

El desarrollo tecnológico de la humanidad, hace cada día posible la integración de nuevos conocimientos a todas las áreas del saber, así como el desarrollo de competencias en diversos campos vinculados tanto a la programación informática como al diseño de ambientes virtuales cada vez más amigables e intuitivos para el fácil manejo de las personas, y por supuesto la comercialización a gran escala de estas propuestas de innovación.

En el ámbito educativo, este desarrollo ha generado grandes cambios, pero al mismo tiempo, enormes desafíos para una generación de docentes que, a pesar de sus años de experiencia, su conocimiento didáctico-pedagógico y su vocación de enseñar; no han sido educados en una época como la actual, en la que las nuevas generaciones nacen con la tecnología en su entorno como algo natural y común. Estas dificultades han acarreado en las últimas décadas diversas posturas por parte del cuerpo docente, que van desde el abandono de la carrera, pasando por la resignación obligada o el entusiasmo aparente, hasta la verdadera capacidad proactiva de adaptarse a la nueva manera de educar.

En este aspecto, el modelo educativo llamado Conectivismo, ha influido positiva o negativamente - no solamente en los docentes, sino también en cada uno de los actores y elementos que componen el currículo, como son los estudiantes, padres de familia, directivos y la institución educativa misma, como el lugar en el que se desarrollan estos cambios, tanto en lo referente a los recursos físicos como a los paradigmas establecidos respecto a los modelos tradicionales. La implementación de modelos educativos basados en el conectivismo, tuvo desde el inicio dificultades en países como el nuestro con un acceso limitado a la tecnología, pero sobre todo por la falta de incentivos para la innovación, sobre todo en el ámbito de la educación pública.

Esta problemática se ha hecho latente de manera más profunda a partir de las necesidades surgidas por la pandemia del COVID 19, dejando al descubierto diferentes realidades socio emocionales, que, ahondadas por el tema sanitario, desembocaron en un sinnúmero de resultados más negativos que positivos, pasando desde la depresión estudiantil, hasta inclusive la deserción escolar en muchos casos, definitiva. 


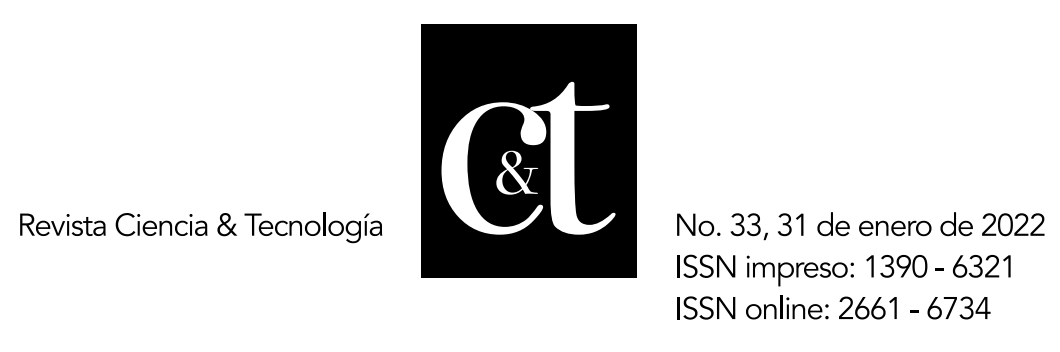

Para (López et al., 2021) tanto las familias como los docentes se vieron en la necesidad de implementar nuevas herramientas y plataformas virtuales sin experiencia previa, lo cual "fue, sin duda, causa de angustia y estrés para docentes, alumnos y seguramente también para sus familiares"(p. 2).

De acuerdo a Velasco Yanez et al. (2021), la salud mental de los adolescentes se vio gravemente afectada debido no solo a la incertidumbre respecto al posible contagio y muerte propia y de familiares, sino también debido al confinamiento obligado, que trastocó su desarrollo social cotidiano, aumentando aceleradamente los porcentajes de inestabilidad y la necesidad de atención psicológica principalmente en las mujeres, como lo muestra la figura 1:

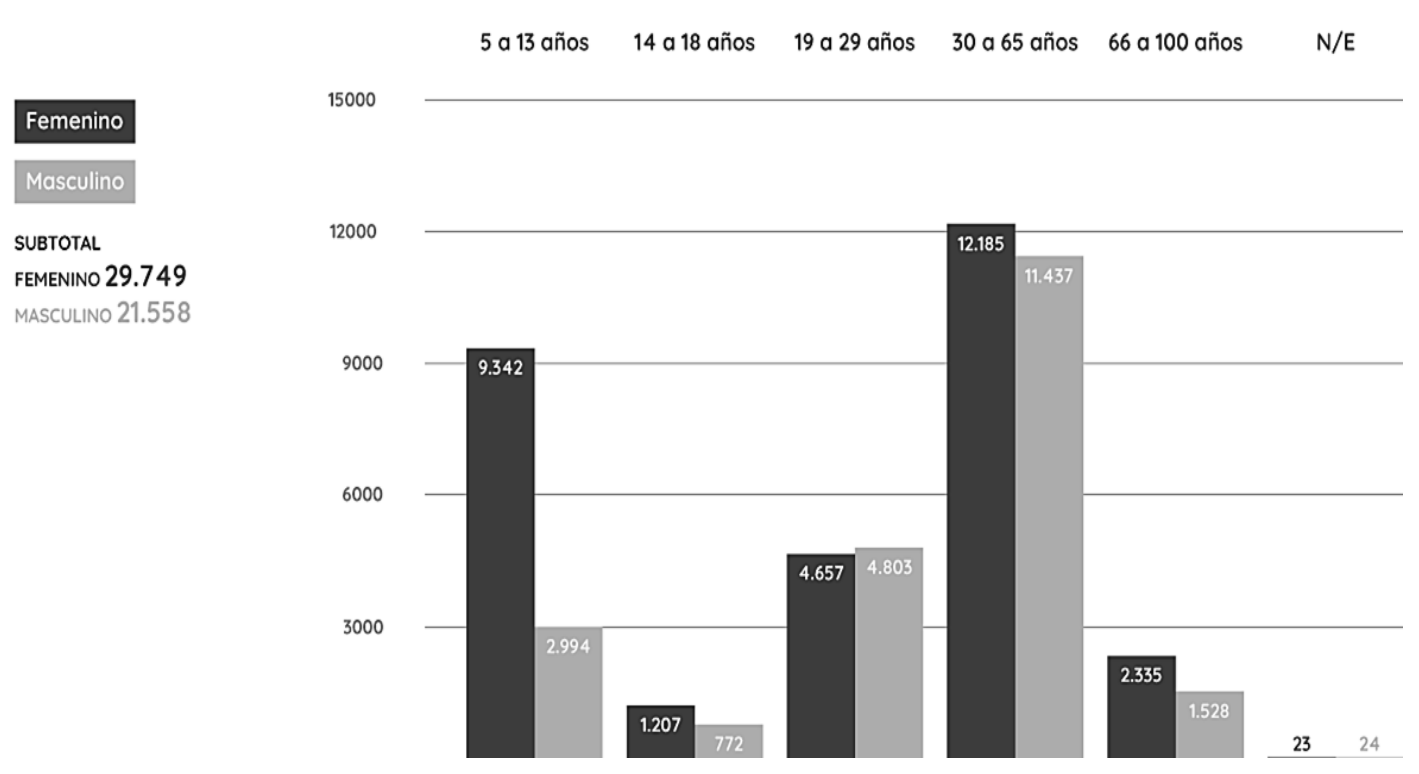

Figura 1: Atenciones de la línea 171 opción 6 del Ministerio de Salud entre mayo y septiembre de 2020

Fuente: Ministerio de Salud del Ecuador (2020)

Estos datos son fundamentales para comprender en toda su magnitud la afectación que tuvo la pandemia, no solamente en lo económico o de salud, sino también en todas las metas planteadas a nivel internacional por los distintos países, que se habían embarcado en proyectos de mejoramiento de la calidad educativa a través de acuerdos y propuestas como los ODS $^{2}$ de la Asamblea General de las Naciones Unidas, que se vieron truncados gravemente.

Conforme a lo descrito por Velasco Yanez et al. (2021) "En el caso de Ecuador, tanto las evaluaciones nacionales como internacionales indicaban que antes de la pandemia los aprendizajes de los alumnos no eran satisfactorios"(p.3),

\footnotetext{
${ }^{2}$ Objetivos de desarrollo sostenible
} 


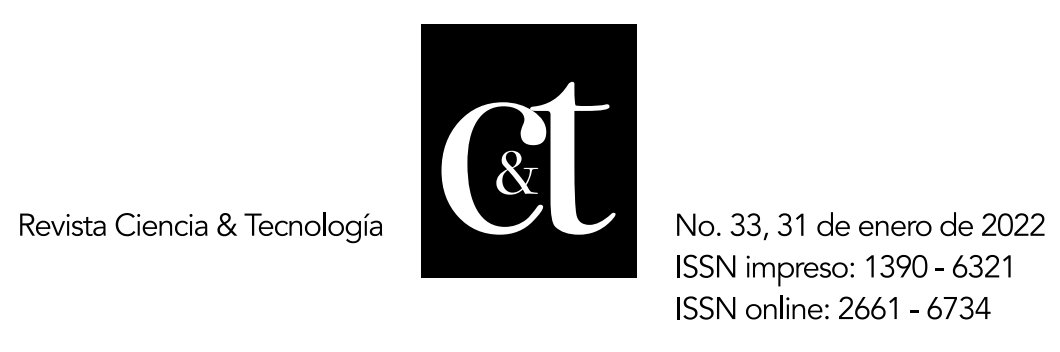

y estas deficiencias se vieron profundizadas y puestas en mayor evidencia con los estragos causados por el COVID 19.

A través de este breve estudio, se intenta describir y analizar desde la perspectiva de varios autores y la interpretación propia basada en la experiencia docente del autor, la problemática de la situación educativa de nuestro país respecto a los diferentes aspectos que conforman nuestra realidad frente a los desafíos del conectivismo, propuesta que en los últimos años se ha colocado a la vanguardia como la opción que, para muchos, permite un desarrollo idóneo de la calidad de los sistemas educativos a nivel mundial.

\section{Desarrollo}

La aparición del Internet en el año 1969, trajo consigo una revolución comunicativa, que jamás volvió a ser la misma, permitiendo abrir un mundo completo de nuevas potencialidades para todos los ámbitos de la sociedad. A nivel educativo, esta revolución vino de la mano con grandes desafíos que a la vez que retaron la creatividad de los actores, permitió un inherente mejoramiento de las capacidades de acceso a la información y al conocimiento. En este marco progresivo de cambios, surgió la teoría conectivista de George Siemens como una alternativa a la educación tradicional, señalando principalmente que el conocimiento se desarrolla en colaboración, a manera de redes interactuando permanentemente entre sí (Nava-Casarrubias, 2014) con la finalidad de compartir conocimientos y desarrollar a la vez habilidades comunicativas más allá del simple uso social de las redes.

De acuerdo a Siemens (2007):

El conectivismo es la integración de principios explorados por las teorías de caos, redes, complejidad y auto-organización. El aprendizaje es un proceso que ocurre al interior de ambientes difusos de elementos centrales cambiantes - que no están por completo bajo control del individuo. El aprendizaje (definido como conocimiento aplicable) puede residir fuera de nosotros (al interior de una organización o una base de datos), está enfocado en conectar conjuntos de información especializada, y las conexiones que nos permiten aprender más tienen mayor importancia que nuestro estado actual de conocimiento. (p. 6)

Estas características establecen la necesidad imperante de medios tanto físicos como virtuales que permitan la integración de diversos entornos para la consecución de los objetivos planteados a través de esta teoría. El constante cambio al que se encuentra sujeto cada uno de los elementos que conforman la base de estos procesos, obligan también a tener una perspectiva claramente fluida, que permita acompañar dichos cambios de una manera resiliente y productiva, dejando atrás el estatismo y la rigidez de otras posturas más convencionales de hacer educación.

La premisa que sustenta el conectivismo reside en la idea de que el potencial de las TIC está alterando nuestros cerebros, puesto que estas herramientas interactivas que utilizamos, en contraste con herramientas digitales más antiguas y más pasivas en penetración, definen y moldean la forma en que 


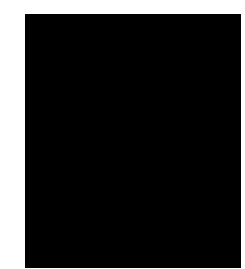

gestionamos la información generando un pensamiento más activo y más rápido. (Recio et al., 2017, p. 4)

Esto implica que la gestión del conocimiento ha pasado a ser en los últimos tiempos una necesidad imperativa que conduce el desenvolvimiento acelerado de las diferentes profesiones, al punto que no existe hoy una sola carrera profesional que no potencie como característica propia el desarrollo de habilidades en el campo tecnológico, en especial porque "muchos de los procesos manejados previamente por las teorías de aprendizaje (en especial los que se refieren al procesamiento cognitivo de información) pueden ser ahora realizados, o apoyados, por la tecnología" (Siemens, 2007, p.2). Según los principios del conectivismo enunciados por Siemens, el aprendizaje a través de redes proporciona la oportunidad de acceder a la información y los conocimientos a través del desarrollo colaborativo de los procesos educativos, permitiendo la toma de decisiones adecuadas para el mejoramiento continuo (Figura 2).

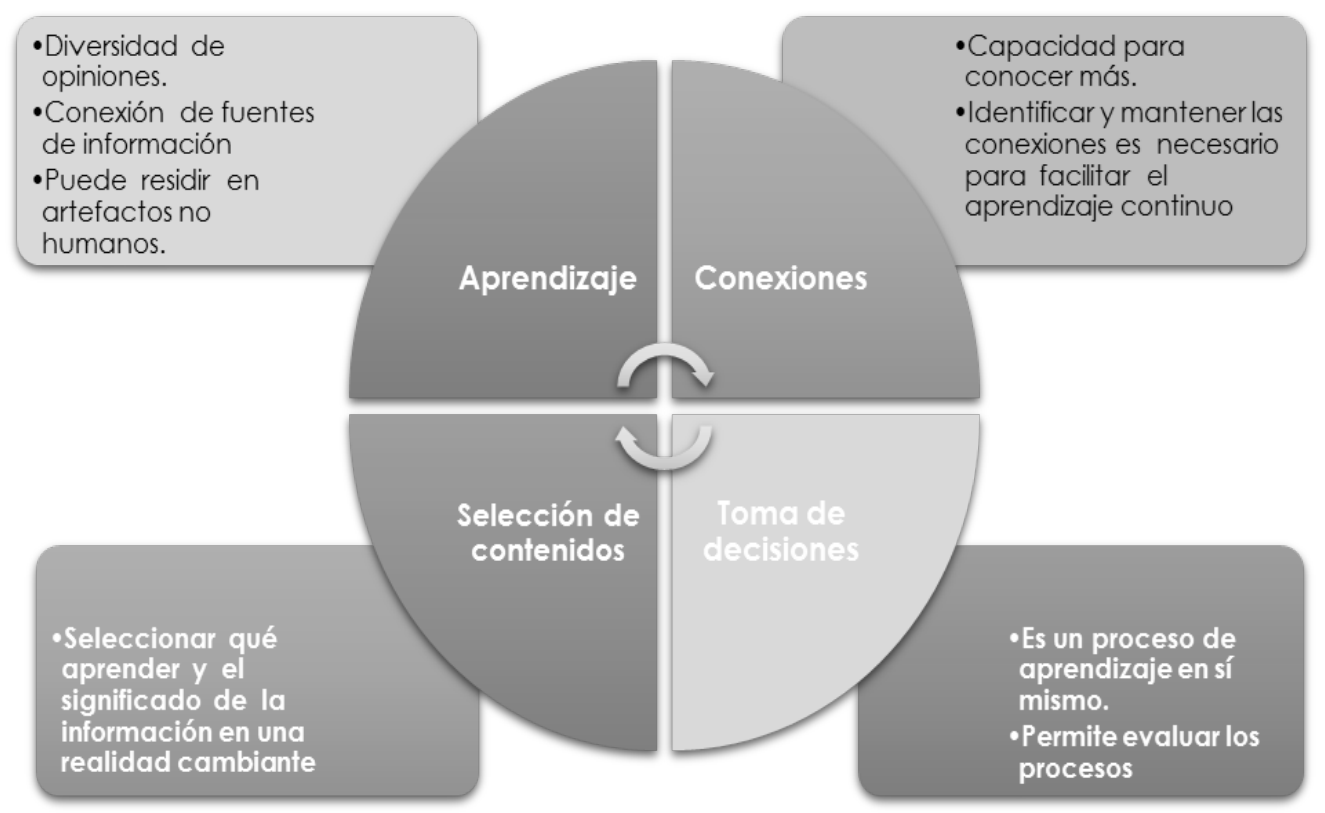

Figura 2: Principios del Conectivismo

Fuente: Elaboración propia basada en (Gutiérrez Campos, 2012)

Estas características, han acarreado a nivel mundial diversas connotaciones, que sobre todo en el campo educativo han sido abordadas de acuerdo a la visión particular de cada país, pero sobre todo a la capacidad de recursos que éstos tengan para el impulso de estos cambios. En países en vías de desarrollo, como el nuestro, existen aún un sinnúmero de dificultades o limitaciones que se deben solventar para una aplicación idónea del modelo conectivista que podemos considerar que se producen desde 3 perspectivas principales: 


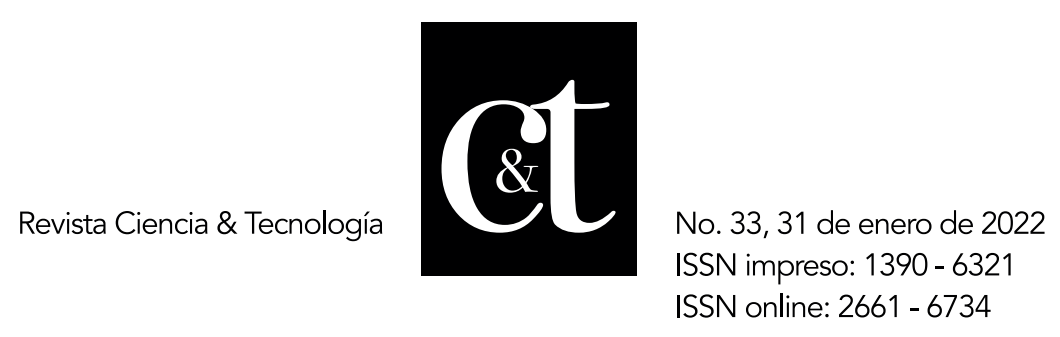

la dificultad de acceso por razones económicas, el tradicionalismo de pensamiento colectivo y la falta de procesos de capacitación docente.

La dificultad de acceso por razones económicas. En un país como el nuestro, en el que los índices de pobreza fluctúan entre el $25 \%$ para el sector urbano, y el $40 \%$ para el sector rural (INEC, 2020), el acceso a los medios tecnológicos se hace complicado para una buena parte de la población, una realidad que es determinante para que las instituciones educativas se abstengan en muchos casos de intentar propuestas de innovación.

La interconexión requiere que los estudiantes puedan acceder desde sus casas a los recursos virtuales de aprendizaje propuestos por los docentes, lo cual es imposible para hogares que se mantienen con el sueldo básico o incluso menos. $\mathrm{Si}$ a esto se suma el hecho de que muchos de los niños que viven en estas situaciones precarias tienen que salir a buscar una forma de subsistir en apoyo de sus padres - o incluso obligados por ellos - nos encontramos con una triste pero muy clara realidad que impide la aplicación de estos tipos de teorías.

De acuerdo a (INEC, 2021), tan solo el $53.2 \%$ de los hogares ecuatorianos tiene acceso a Internet, como se muestra en la figura 3, lo cual ejemplifica la amplia brecha digital que existe en nuestra sociedad, que no dista mucho de la de la mayoría de países latinoamericanos.

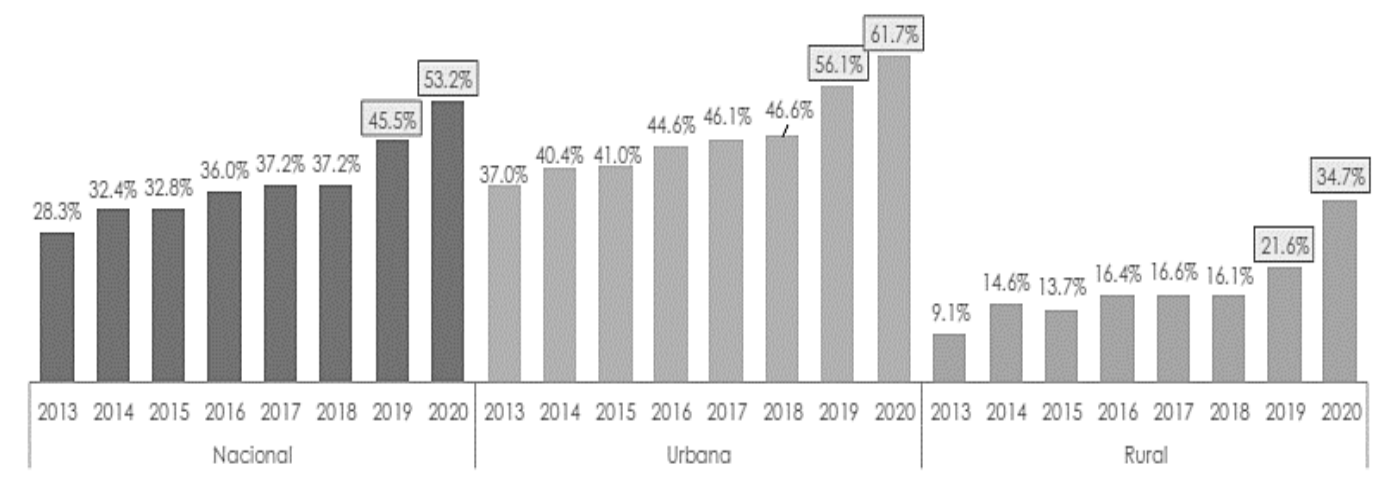

Figura 3: Hogares con acceso a Internet

Fuente: (INEC, 2021)

Ante el aislamiento social obligatorio, los procesos educativos en todo el país tuvieron que reajustarse a la nueva modalidad online o e-learning; desnudando las principales carencias de nuestro sistema educativo en el campo de la implementación tecnológica incluso desde el mismo Ministerio de Educación, hasta llegar a prácticamente todas las instituciones públicas, que son víctimas de esa falta de recursos a la hora de intentar establecer la conexión entre estudiantes, maestros y directivos.

El tradicionalismo de pensamiento colectivo. Aunque a través de los años, se ha intentado desarraigar los pensamientos netamente conductistas, repetitivos y memoristas de una sociedad que aún replica modelos tradicionales de 
educación, en la práctica, existe todavía una clara tendencia a volver a los procesos educativos antiguos, los cuales - sin desmerecer muchos de ellos que dieron resultado para su época - ya no son necesariamente los más idóneos, por lo menos no de forma directa y aislada, para generar comprensiones profundas y aplicabilidad de los conocimientos de forma significativa en nuestra realidad.

Existe una tendencia a pensar que "todo lo pasado fue mejor", generalizando un tradicionalismo colectivo que abarca a padres de familia, directivos e incluso maestros que, ante la aparente falta de resultados de los modelos actuales, sobre todo en lo referente a las competencias académicas vinculadas al desarrollo profesional y de investigación; crean una ola de pensamiento que en muchos casos coge fuerza para desechar y desmerecer los procesos vinculados a la teorías tecnológicas de la educación.

En muchos casos, este rechazo puede provenir también de la Tecnofobia que según Caballero (2013) citado por Hervás-Gómez et al. (2016) se define como el "miedo o aversión hacia las nuevas tecnologías o dispositivos complejos" ( $p$. 87). Este temor, impulsa un ensimismamiento del docente hacia lo tradicional de su actuar, provocando el bloqueo hacia los nuevos modelos, y estancando su evolución, hasta el punto de inclusive hacerle perder su trabajo. Por otro lado, existen quienes adoptan los modelos conectivistas impulsados por el afán de conseguir mejores resultados, pero de una manera descoordinada entre tecnología y pedagogía, obteniendo resultados que tampoco son los esperados.

De acuerdo a Ramírez (2016), parte de la culpa del fracaso proviene de la mala aplicación de los modelos pedagógicos, incluso del constructivismo, que aunque en su planteamiento se ha abierto necesariamente al uso de las TIC como medio idóneo para la construcción de los aprendizajes, "...en muchos casos la implementación del constructivismo se reduce a prácticas pedagógicas que mucho tienen de tradicionales y muy poco de innovadoras" (p. 5), confundiendo de esta manera la visión que se tiene respecto al uso educativo de las tecnologías.

Esta situación se ahonda debido a las realidades sociales que se viven también en el aspecto disciplinario, cuyos resultados lastimosamente están a la vista diariamente con la proliferación de las redes sociales, que muestran una pérdida total del respeto hacia los docentes por parte de estudiantes y padres de familia; lo cual no es un problema necesariamente generado desde la educación formal, sino más bien una pérdida de valores generada en el hogar y alimentada por la proliferación de ideologías que relativizan todas las formas de pensamiento formales.

Resulta entonces muy fácil culpar erróneamente de esto a los modelos actuales de hacer educación, y provocar así un rechazo colectivo al uso de las tecnologías o al menos una reticencia por parte de las generaciones adultas.

La falta de procesos de capacitación docente. De acuerdo a Recio et ál. (2017) los profesores no están todavía debidamente preparados para el cambio hacia el conectivismo, ya que necesitan ser capacitados no solamente en tecnologías, 


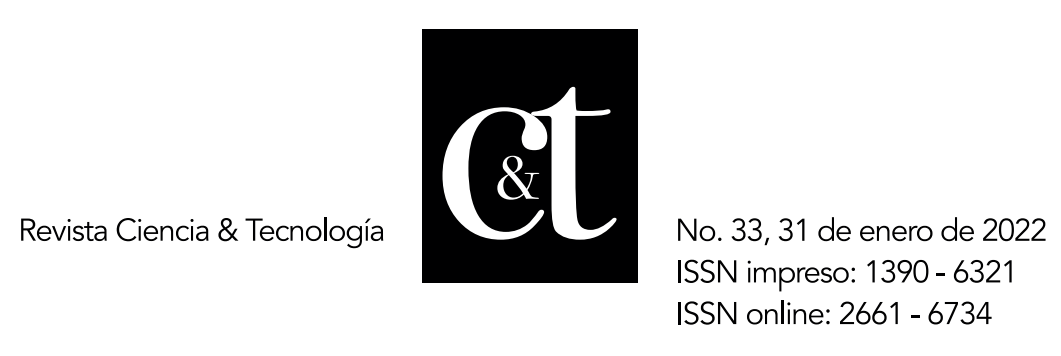

sino en un cambio de su perspectiva pedagógica para comprender las implicaciones del modelo.

Para Cueva Delgado et al. (2020), "se necesitan docentes de estos tiempos, que evolucionen, que sean capaces de integrar el uso de los medios tecnológicos en sus procesos de enseñanza aprendizaje y no convertirse en meros trasmisores de información" (p. 7), sino creadores de ambientes activos de aprendizaje, que a la vez tengan coherencia curricular, y no se desliguen de los procesos cognitivos básicos que los estudiantes deben desarrollar. Un reto para lo cual se requiere de una constante actualización de conocimientos, tanto tecnológicos, como didácticos que le brinden la base epistemológica necesaria para ponerla en práctica.

Es importante mencionar que esta dimensión no se presenta como desventaja, debido necesariamente a que no existan propuestas de capacitación en el ámbito de las tecnologías educativas, ya que como podemos ver actualmente la demanda de estos temas y la cantidad de empresas y personas que ofertan este tipo de cursos ha aumentado exponencialmente. Pero esto no necesariamente asegura ni la calidad, ni la contratación de dichos cursos por parte de los maestros.

La realidad económica del docente ecuatoriano toma parte en este aspecto, ya que en muchos de los casos el sueldo no es suficiente para solventar estos cursos que en su mayoría tienen un costo que, sin ser necesariamente muy elevado, no siempre se encuentran al alcance de los docentes tanto en horarios como en valor económico. Si a esto le sumamos la apatía reinante respecto a la actualización por parte de docentes extenuados con la carga de trabajo administrativo que hoy en día se le añade a la ya de por sí exigente labor pedagógica - diseño de planes, recursos, materiales, evaluaciones - nos encontramos con un panorama que impide la preparación académica idónea de nuestros profesores.

\section{Conclusiones}

Aunque el Conectivismo es una tendencia real que se propone como camino obligatorio hacia la calidad de los procesos educativos, nuestro sistema escolar aún presenta muchas dificultades desde el punto de vista de acceso tecnológico, así como en lo referente a la infraestructura y los procesos pedagógicos que se requieren para la implementación de este tipo de modelos.

A partir de la pandemia, hubo avances significativos en los procesos de capacitación docente a nivel de herramientas tecnológicas, aunque no se pueden considerar suficientes todavía, ya que se requiere además de un cambio paradigmático que, desde una nueva concepción de la Educación, promueva los avances reales a nivel de los procesos de aprendizaje, en los que todos los actores se comprometan a poner su aporte real en las innovaciones desde diferentes ámbitos.

La realidad ecuatoriana dista mucho aún del ideal requerido para estas innovaciones, ya que los recursos asignados no son suficientes para producir los cambios necesarios. 


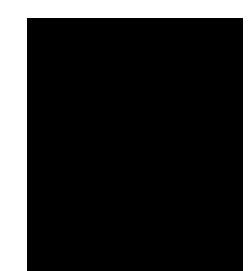

Se torna urgente un cambio drástico en las políticas públicas para generar una verdadera revolución de nuestro sistema educativo, que tienda a valorar el trabajo docente, permitiendo el acceso a la capacitación real y no solamente al cumplimiento obligatorio de requisitos. Esto derivará en una revalorización por parte de la sociedad, recuperando el respeto hacia la profesión docente y el desarrollo de las normas y valores intrínsecos del ser humano.

Por otro lado, la inversión en el mejoramiento de la infraestructura y los recursos tecnológicos necesarios para la interconexión y la formación de redes de aprendizaje colaborativo, producirán un mejoramiento continuo y real de la calidad en nuestra educación, haciendo visible la evolución académica ante el mundo.

«Cuando el aprendizaje y la enseñanza mejoran continuamente, todo mejora»

Nelson Mandela

\section{Referencias Bibliográficas}

Cueva Delgado, J. L., García Chávez, A., \& Martínez Mooina, O. A. (2020). La influencia del conectivismo para el uso de las TIC en el proceso enseñanzaaprendizaje. Revista Dilemas Contemporáneos: Educación, Política y Valores, 7(2), 54-67. https://doi.org/https://doi.org/10.46377/dilemas.v32i1.1975

Gutiérrez Campos, L. (2012). Conectivismo como teoría de aprendizaje: conceptos, ideas, y posibles limitaciones. Revista Educación y Tecnología, 1, 111-122. file://C:/Users/CalasanzParaTodos/Downloads/DialnetConectivismoComoTeoriaDeAprendizaje-4169414.pdf

Hervás-Gómez, C., López Mata, E., Real Plehan, S., \& Fernández Márquez, E. (2016). Tecnofobia: competencias, actitudes y formación del alumnado del Grado en Educación Infantil. IJERI: International Journal of Educational Research and Innovation, 6, 83-94.

https://idus.us.es/bitstream/handle/11441/68868/Tecnofobia competencias, actitudes y formación del alumnado del Grado en Educación Infantil.pdf?sequence $=1$

INEC. (2020). Boletín técnico de empleo enemdu septiembre 2020. In Instituto Nacional de Estadística y Censos. https://www.ecuadorencifras.gob.ec/documentos/webinec/Estadisticas_agropecuarias/espac/espac-2018/Boletin tecnico.pdf

INEC. (2021). Tecnologías de la Información y Comunicación , 2020. In Instituto de la Información y Comunicación. https://www.ecuadorencifras.gob.ec/documentos/webinec/Estadisticas_Sociales/TIC/2020/202012_Principales_resultados_Multiproposito _TIC.pdf

López, M., Herrera, M., \& Apolo, D. (2021). Educación de calidad y pandemia: retos, experiencias y propuestas desde estudiantes en formación docente de Ecuador. Sustainable Development of Mountain Territories, 14(2), 1-12. https://doi.org/10.35699/1983-3652.2021.33991

Nava-Casarrubias, A. (2014). Hacia una educación conectivista. August. 


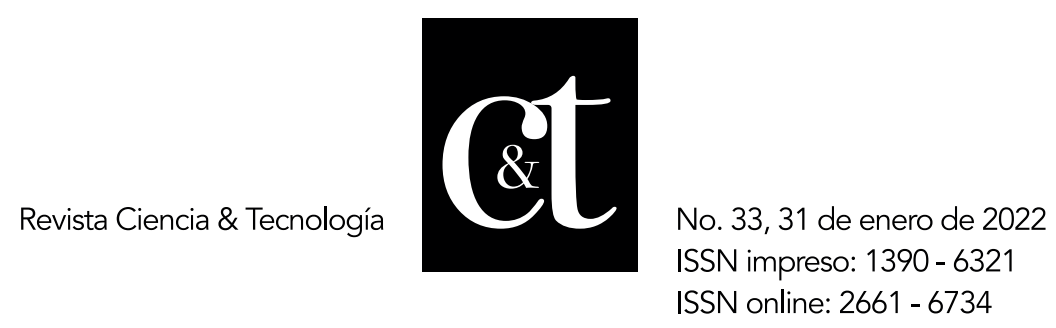

Ramírez, R. (2016). Conectivismo y entornos personales de aprendizaje en la dinámica pedagógica del siglo XXI. I Congreso Internacional Interdisciplinariedad y Desarrollo.

https://d1wqtxts1xzle7.cloudfront.net/53178828/EL_CONECTIVISMO_Y_LOS_ENT ORNOS_PERSONALES_DE_APRENDIZAJE-with-cover-page-

v2.pdf?Expires $=1634866329 \&$ Signature $=$ Uve $\sim$ M3-

Rqm0asUYi7zDEBZ81dabMQIQrakdhORh7UI2L67fYuvfN4QR8T3qV4025rSMhYG8pJd 0T8ZL-244mxGKVDGieaqq0BPY

Recio, C., Díaz, J., \& Saucedo Fernández, M. (2017). Conectivismo, ventajas y desventajas. In VII Congreso Virtual Iberiamericano de Calidad en Educación Virtual y a Distancia (14th ed.).

Siemens, G. (2007). Conectivismo: Una teoría de aprendizaje para la era digital. Comenius, 5, 1-10. https://skat.ihmc.us/rid=1J134XMRS-1ZNMYT4-13CN/George Siemens - Conectivismo-una teoría de aprendizaje para la era digital.pdf

Velasco Yanez, R., Cunalema Fernández, J., Franco Coffre, J., \& Vargas Aguilar, G. (2021). Estrés percibido asociado a la pandemia por COVID-19 en la ciudad de Guayaquil, Ecuador. Ministerio Del Poder Popular Para La Salud de Malariología y Salud Ambiental, LXI(1), 38-46.

https://docs.bvsalud.org/biblioref/2021/04/1177559/art-2-i-2021.pdf 


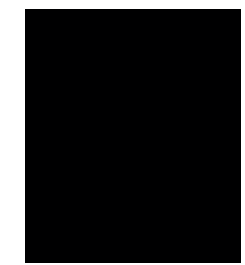

Revista Científica Ciencia y Tecnología Vol 22 No 33 págs. 80-89 http://cienciaytecnologia.uteg.edu.ec 
No. 33, 31 de enero de 2022

ISSN impreso: 1390 - 6321

ISSN online: 2661 - 6734 